\title{
The Closed-loop Identification Problem in Indirect Adaptive Control
}

\author{
J.W. Polderman \\ Department of Applied Mathematics, \\ University of Twente \\ P.O. Box 217 \\ 7500 AE Enschede, The Netherlands. \\ C. Praagman \\ Department of Mathernatics and Computing Science \\ University of Eindhoven \\ P.O. Box 513
}

5600 MB Eindhoven, The Netherlands.

\begin{abstract}
It is well known that in indirect adaptive control algorithms, the system to be controlled is not necessarily identified due to lack of excitation caused by the fact that identification takes place in closed-loop. Whether or not this leads to undesirable behaviour depends on the underlying control criterion.

A classification is made of all control criteria for which the closed-loop identification problem does not obstruct the desired controlled behaviour of the system. Under mild assumptions it is proved that these criteria are all of the poleassignment type.
\end{abstract}

\section{Introduction}

The problem we want to address is the following. For which adaptive control problems do we have the property that, despite the fact that, due to identification in closed-loop, the system to be controlled is not identified, the system is controlled properly. More precisely, we consider adaptive control algorithms of the following type. Estimates of the system parameters are made on the basis of the observed input/output behaviour. Based on the estimate the controller parameters are adjusted as if the estimate correctly describes the system (certainty equivalence). After having applied the resulting control input, the behaviour of the system is compared with the expected behaviour and if they do not agree, a new estimate is produced (identification driven by prediction error). This type of algorithm is sometimes referred to as indirect adaptive control based on certainty equivalence

and driven by the prediction error. This class of algorithms has the feature that other parameters than the ones that describe the open-loop behaviour of the system, cause zero prediction error for the closed-loop system. A first reaction could be that this is undesirable: bad estimates will give rise to bad controllers. On the other hand, due to some miracle, a wrong estimate could coincidently give the right controller. Surprisingly the latter possibility indeed occurs in some adaptive control problems.

This unexpected phenomenon has been reported in the literature at several places. Probably the first account is [2], where it was observed that for the minimum variance controller, the sequence of estimates converged to the wrong value, yet inducing optimal behaviour asymptotically. A rigorous treatment of this effect for the minimum variance controller was given in [3]. Independently similar effects were reported implicitly in several papers dealing with adaptive pole-assignment see $[1,4,5,6,8,11,14]$. A complete analysis of this problem is provided in $[12,14])$.
Accounts of the opposite effect, wrong estimates induce wrong controllers are $[10,13]$, where adaptive $L Q$ control was investigated. The simple conclusion could be that some adaptive controllers suffer from identification in closed-loop and others do not. Rather than acquiesce this laconic viewpoint, one could try to predict in advance to which of the two categories a specific adaptive control problem belongs.

In [12] a first attempt was made to classify all adaptive control problems for which zero prediction error is equivalent to desired behaviour. The problem was solved only partially. In the present paper a next step towards a classification is made, although a complete solution has not yet been found.

The paper is organised as follows. In the next section we give the precise problem statement. In the third section the classification problem is completely solved for the observed-state case. The fourth section deals with the more realistic case that the state is not observed directly. Finally, in the last section, we draw some conclusions. Because of space limitations, we do not provide all the proofs. For the complete proofs, the reader is referred to [15]

\section{Problem Statement}

The class of systems we consider is the class of single- input/singleoutput systems of order $n$, represented in state space form. All systems are assumed to be deterministic and disturbance-free. We will use the following notation:

$$
\begin{aligned}
& E:=\left\{(A, b, c) \in \mathbb{R}^{n \times n} \times \mathbb{R}^{n \times 1} \times \mathbb{R}^{1 \times n} \mid(A, b, c) \text { minimal }\right\} \\
& E_{r e}:=\left\{(A, b) \in \mathbb{R}^{n \times n} \times \mathbb{R}^{n \times 1} \mid(A, b) \text { reachable }\right\}
\end{aligned}
$$

With $(A, b, c) \in E$ we associate the system:

$\left\{\begin{aligned} x_{k+1} & =A x_{k}+b u_{k} \\ y_{k} & =c x_{k}\end{aligned}\right.$

We only consider control problems for which for every system $(A, b, c)$ there exists a unique control-law $u_{k}=f x_{k}$ that solves the problem. The control-law $f$ is thus a function of the parameters $(A, b, c)$. We will have to make assumptions on how $f$ depends on $(A, b, c)$, but first we will explain that whether or not wrong estimates induce good behaviour depends solely on $f$. In order to do that we do not have to refer to a specific identification method since we will only study the asymptotic behaviour, loosely speaking the behaviour after the sequence of estimates has converged. On that level of generality the only thing we know is that estimates 
converge, if at all, to a value for which the prediction error is zero. Hence we have to compare the behaviour of the true system on the one hand and the behaviour of the estimate on the other. Let the true system be represented by $\left(A_{0}, b_{0}, c_{0}\right) \in E$, and the estimate by $\left(A, b, c_{0}\right) \in E$. Note that by changing the basis of the state space, the estimate of $c_{0}$ can always be taken $c_{0}$. We then have:

$$
\left\{\begin{aligned}
x_{k+1} & =A_{0} x_{k}+b_{0} u_{k} \\
y_{k} & =c_{0} x_{k} \\
z_{k+1} & =A z_{k}+b u_{k} \\
\hat{y}_{k} & =c_{0} z_{k}
\end{aligned}\right.
$$

The input applied to both the true system and the model is, according to the certainty equivalence principle:

$u_{k}=f\left(A, b, c_{0}\right) z_{k}$

Where $f: E \rightarrow \mathbb{R}^{1 \times n}$ is the function that assigns to every system $(A, b, c) \in E$ a control-law. We assume that the criterion that lies at the basis of the control-law $f$, is a criterion on the input/output behaviour of the system. As a consequence the input $u_{k}=f(A, b, c) z_{k}$ should not depend on the choice of basis in the state-space. Therefore it follows that $f$ satisfies the transformation property:

$\forall S \in G l(n): f\left(S A S^{-1}, S b, c S^{-1}\right)=f(A, b, c) S^{-1}$

$\left(A, b, c_{0}\right)$ is an invariant point of any identification scheme if and only if:

$\forall k: \hat{y}_{k}=y_{k}$

Note that the state-sequences $\left\{x_{k}\right\},\left\{z_{k}\right\}$ are uniquely determined by (4) and (7).

Our main concern in this paper is the classification of all functions $f$ that have the following property:

Property 2.1 The equations $(4,5,7)$ imply that for all $k$ :

$u_{k}=f\left(A, b, c_{0}\right) z_{k}=f\left(A_{0}, b_{0}, c_{0}\right) x_{k}$

If $f$ satisfies this property, then indeed every invariant point of the algorithm produces the desired input sequence, despite the fact that $\left(A_{0}, b_{0}, c_{0}\right)$ might not be the only invariant point.

For the readers convenience we will now give an example of a feedback-law $f$ that has this surprising property.

Theorem 2.2 Let $\pi \in \mathbb{R}[\boldsymbol{X}]$ be a monic polynomial of degree $n$. Define $f: E \rightarrow \mathbb{R}^{1 \times n}$ by:

$f(A, b, c)=-[0 \ldots 01]\left[b \vdots \ldots \vdots A^{n-1} b\right]^{-1} \pi(A)$

For every $\left(A_{0}, b_{0}, c_{0}\right),\left(A, b, c_{0}\right)$ satisfying $(4,5,7)$ we also have $(8)$.

Remark 2.3 The function $f$ in Theorem 2.2 is the feedback-law that assigns the closed-loop poles to the roots of the polynomial $\pi$. It is known as Ackermann's formula (see (7)).

The proof of Theorem 2.2 is based on the following two results:

Theorem 2.4 Let $\left(A_{0}, b_{0}, c_{0}\right),\left(A, b, c_{0}\right) \in E$ and $\mathrm{V}$ a linear subspace of $\mathbb{I R}^{n \times n}$ such that for all $v \in \mathrm{V}$ :

(i) $\left(A_{0}+b_{0} f\left(A, b, c_{0}\right)\right) v \in \mathbf{V}$

(ii) $\left(A_{0}+b_{0} f\left(A, b, c_{0}\right)\right) v=\left(A+b f\left(A, b, c_{0}\right)\right) v$

If $f$ is of the form (9), then:

for all $v \in \mathbf{V}: f\left(A, b, c_{0}\right) v=f\left(A_{0}, b_{0}, c_{0}\right) v$.
Theorem 2.5 Let $\left\{\left(u_{k}, y_{k}\right)\right\}_{k \in \mathrm{N}}$ be a sequence in $\mathbb{R}^{2}$ and suppose there exist $\left(A_{1}, b_{1}, c_{1}\right),\left(A_{2}, b_{2}, c_{2}\right) \in E$, and sequences $\left\{x_{k}^{(1)}, x_{k}^{(2)}\right\}$ in $\mathbb{R}^{n}$, such that for all $k$ :

$\left\{\begin{aligned} x_{k+1}^{(1)} & =A_{1} x_{k}^{(1)}+b_{1} u_{k} \\ y_{k} & =c_{1} x_{k}^{(1)} \\ x_{k+1}^{(2)} & =A_{2} x_{k}^{(2)}+b_{2} u_{k} \\ y_{k} & =c_{2} x_{k}^{(2)}\end{aligned}\right.$

Define $\mathbf{X}_{i}=\operatorname{span}\left(\left\{x_{k}^{(i)}\right\}_{k \in \mathbf{N}}\right)$, and $d_{i}=\operatorname{dim}\left(\mathbf{X}_{i}\right), i=1,2$.

1. if $d_{1}<n$, then there exists a non-singular matrix $S$, such that: $S x_{k}^{(1)}=x_{k}^{(2)}$.

2. $d_{1}=d_{2}$

3. if there exists a $g_{1}$ such that: $u_{k}=g_{1} x_{k}^{(1)}$, then there exists a non-singular matrix $S$, such that: $S x_{k}^{(1)}=x_{k}^{(2)}$.

For the proofs of Theorems 2.2, 2.4 and 2.5, the reader is referred to $[12]$.

We will now give necessary and sufficient conditions for the function $f$ to satisfy Property 2.1 .

Theorem 2.6 The feedback-law $f$ satisfies Property 2.1 if and only if for all $c_{0} \in \mathbb{R}^{1 \times n},\left(A_{0}, b_{0}, c_{0}\right),\left(A, b, c_{0}\right) \in E$ and for all subspaces $\mathrm{V}$ of $\mathbb{I R}^{n}$ such that:

$\left.\left(A_{0}+b_{0} f\left(A, b, c_{0}\right)\right)\right|_{\mathrm{v}}=\left.\left(A+b f\left(A, b, c_{0}\right)\right)\right|_{\mathrm{v}}$

$\left(A_{0}+b_{0} f\left(A, b, c_{0}\right)\right) \mathbf{V} \subset \mathbf{V}$

the following holds:

$\left.f\left(A, b, c_{0}\right)\right|_{\mathrm{v}}=f\left(A_{0}, b, c_{0}\right) \mid \mathrm{v}$

Proof If. Choose $\left(A_{0}, b_{0}, c_{0}\right),\left(A, b, c_{0}\right)$ such that $(4,5,7)$ hold. By Theorem 2.5.3 there exists a non-singular matrix $S$ such that for all $k$ :

$S z_{k}=x_{k}$

Note that $(7,17)$ imply that $S$ can be chosen such that:

$c_{0} S^{-1}=c_{0}$

This yields two recursions for $x_{k}$ :

$$
\begin{aligned}
& \begin{aligned}
x_{k+1} & =A_{0} x_{k}+b_{0} f\left(A, b, c_{0}\right) z_{k} \\
& =\left(A_{0}+b_{0} f\left(A, b, c_{0}\right)\right) S^{-1} x_{k} \\
& =\left(A_{0}+b_{0} f\left(S A S^{-1}, S b, c_{0}\right)\right) x_{k}
\end{aligned} \\
& \text { and: } \\
& \begin{aligned}
x_{k+1} & =S z_{k+1} \\
& =S\left(A+b f\left(A, b, c_{0}\right)\right) z_{k} \\
& =\left(S A S^{-1}+S b f\left(S A S^{-1}, S b, c_{0}\right)\right) x_{k}
\end{aligned}
\end{aligned}
$$

Define $(\bar{A}, \hat{b})=\left(S A S^{-1}, S b\right)$ and $\mathrm{V}=\operatorname{span}\left(\left\{x_{k}\right\}\right.$, then it follows from the two recursions $(19,20)$ that:

$\left(A_{0}+b_{0} f\left(\bar{A}, \bar{b}, c_{0}\right)\right)\left|\mathrm{v}=\left(\bar{A}+\bar{b} f\left(\bar{A}, \bar{b}, c_{0}\right)\right)\right| \mathrm{v}$

Also it is not difficult to see that:

$\left(A_{0}+b_{0} f\left(\bar{A}, \bar{b}, c_{0}\right)\right) \mathrm{V} \subset \mathrm{V}$

Since by assumption, $(14,15)$ implies $(16)$ it follows that:

$f\left(\bar{A}, \bar{b}, c_{0}\right)\left|\mathrm{v}=f\left(A_{0}, b_{0}, c_{0}\right)\right| \mathrm{v}$

In particular: 
$f\left(\bar{A}, \bar{b}, c_{0}\right) x_{k}=f\left(A_{0}, b_{0}, c_{0}\right) x_{k}$

and hence:

$f\left(A, b, c_{0}\right) z_{k}=f\left(A_{0}, b_{0}, c_{0}\right) x_{k}$

Only if. Let $\left(A, b, c_{0}\right),\left(A_{0}, b_{0}, c_{0}\right), V$ satisfy $(14,15)$. Choose $x_{0} \in$ V. Then:

$x_{k+1}=\left(A_{0}+b_{0} f\left(A_{0}, b_{0}, c_{0}\right)\right) x_{k}$

$=\left(A+b f\left(A_{0}, b_{0}, c_{0}\right)\right) x_{k}$

And hence we can take $z_{k}=x_{k}$ in (4), yielding $y_{k}=\hat{y}_{k}$. From Property 2.1 it follows that for all $k$ :

$f\left(A_{0}, b_{0}, c_{0}\right) x_{k}=f\left(A, b, c_{0}\right) x_{k}$

Since $\left\{x_{0}\right\} \in \mathrm{V}$ was arbitrary it follows that indeed:

$\left.f\left(A_{0}, b_{0}, c_{0}\right)\right|_{\mathrm{v}}=\left.f\left(A, b, c_{0}\right)\right|_{\mathrm{v}}$

Having established Theorem 2.6, the question that we want to answer is: Classify all functions $f: E \rightarrow \mathbb{R}^{1 \times n}$ for which $(14,15)$ implies (16). However, since our analysis is not yet complete, the approach in this paper could very well be characterized as the investigation of the question if there are other control-laws than those of the form (9), and which additional assumptions on $f$ do we have to impose to exclude them.

\section{Classification I: Observed State}

In this section we will consider the case where the state of the system is directly measurable. In that case the function $f$ does not depend on the output vector $c$. It will turn out that under rather mild assumptions the functions that satisfy 2.1 are all of the type (9). Recall that the standing assumptions on $f$ are (6) and ' $(14,15)$ implies (16)'. Since in this section $f$ does not depend on $c$ we will consider $f$ as a function of the variables $(A, b)$ only. Also it appears that it suffices to take $\mathrm{V}=\mathbb{R}^{n}$ in Theorem 2.6. Hence the basic assumptions on $f$ are:

1. For all $\left(A_{1}, b_{1}\right) \in E_{\text {re }}$ and all $\left(A_{2}, b_{2}\right) \in E_{\text {re }}$ :

$$
\begin{aligned}
& A_{1}+b_{1} f\left(A_{2}, b_{2}\right)=A_{2}+b_{2} f\left(A_{2}, b_{2}\right) \Rightarrow \\
& f\left(A_{1}, b_{1}\right)=f\left(A_{2}, b_{2}\right)
\end{aligned}
$$

2. $\forall S \in G l(n), \forall(A, b,) \in E_{\text {re }}$ :

2. $f\left(S A S^{-1}, S b\right)=f(A, b) S^{-1}$

The question we would like to address is the following:

For which functions $f: E_{\text {re }} \rightarrow \mathbb{R}^{1 \times n}$ do we have properties (29) and $(30)$ ?

To give a complete answer to this question we will make two more assumptions:

3. $f$ is continuous on $E_{\text {re }}$.

4. There exists $\left(A_{0}, b_{0}\right) \in E_{\text {re }}$ such that:

$$
\operatorname{Spec}\left(A_{0}+b_{0} f\left(A_{0}, b_{0}\right)\right) \cap \operatorname{Spec}\left(A_{0}\right)=\emptyset
$$

\section{Comment}

3. Continuity is a natural assumption.

4. Assumption (32) is a technical assumption. It means that there is at least one system such that the open-loop poles differ completely from the closed-loop poles of the controlled system. A sufficient condition for this property to hold, is that $f$ is a stabilizing control-law.

$\square$

We can now give a complete characterization of all functions $f$ satisfying assumptions (29) through (32). It will turn out that they are all of the pole-assignment type.

Theorem 3.1 Let $f$ be such that assumptions (29) through (32) are satisfied. There exists a monic polynomial $\pi$, of degree $n$ such that for all $(A, b) \in E_{\text {re }}$ :

$f(A, b)=-[0 \ldots 01] \cdot\left[b \vdots \ldots \vdots A^{n-1} b\right]^{-1} \pi(A)$

Proof (Outline) Fix $\left(A_{0}, b_{0}\right)$ such that assumption (32) holds. Let $\pi_{0}$ be the characteristic polynomial of $\left(A_{0}+b_{0} f\left(A_{0}, b_{0}\right)\right)$. Define:

$G_{0}:=\left\{(A, b) \in E_{\text {re }} \mid A_{0}+b_{0} f(A, b)=A+b f(A, b)\right\}$

and:

$\bar{G}_{0}:=\left\{(A, b) \in E_{r e} \mid A_{0}+b_{0} f\left(A_{0}, b_{0}\right)=A+b f\left(A_{0}, b_{0}\right)\right\}$

Then, by (29), $G_{0}=\bar{G}_{0}$. Choose $(A, b) \in G_{0}$, then, again by (29): $A_{0}+b_{0} f\left(A_{0}, b_{0}\right)=A+b f(A, b)$

Hence the characteristic polynomial of $(A+b f(A, b))$ is equal to $\pi_{0}$. By the uniqueness of the control law that assigns the poles, it follows that for all $(A, b) \in G_{0}$ :

$f(A, b)=-[0 \ldots 01] \cdot\left[b: \ldots \vdots A^{n-1} b\right]^{-1} \pi_{0}(A)$

We will now show that $f$ has the form (37) for all pairs $(A, b)$ that are similar to a pair in $G_{0}$. Choose $S \in G l(n)$, then:

$$
\begin{aligned}
& f\left(S A S^{-1}, S b\right)=f(A, b) S^{-1} \\
& =-[0 \ldots 01] \cdot\left[b \vdots \ldots \vdots A^{n-1} b\right]^{-1} \pi_{0}(A) S^{-1} \\
& =-[0 \ldots 01] .\left[S b: \ldots \vdots\left(S A S^{-1}\right)^{n-1} S b\right]^{-1} \pi_{0}\left(S A S^{-1}\right)
\end{aligned}
$$

Hence for every pair that is similar to a pair in $G_{0}$, we have established the desired formula. It can be shown ([15]) that the set of all pairs that can be reached by a similarity transformation on the elements of $G_{0}$ is open and dense in $E_{r e}$. The continuity of $f$ then yields the result.

We have now established a characterization of all feedback laws, depending only on $(A, b)$, for which Property 2.1 holds. In the next section we will try to extend this classification result to the more general case where the function $f$ may also depend on the c.vector.

\section{Classification II: Unobserved State}

In this section we will consider the general case where $f$ also depend on $c$. The main tool of the analysis in the previous section was the determination of $f$ on the set $G_{0}$ followed by the extension of $f$ to $E_{\mathrm{re}}$ by performing similarity transformations on the elements of $G_{0}$. Since in $(14,15) c_{0}$ is fixed, we now may only apply similarity transformations that leave $c_{0}$ invariant. As a consequence we cannot reach 'almost every point' in $E$, and hence the proof of Theorem 3.1 does not apply here.

Without additional assumptions we have a complete result for the first order case only, which we will present first. 
Theorem $4.1(n=1)$ Let $f: \mathbb{R} \times \mathbb{R} \backslash\{0\} \times \mathbb{R} \backslash\{0\} \rightarrow \mathbb{R}$ be such that Property 2.1 holds. Assume that there exists $\left(a_{1}, b_{1}\right)$ and $\left(a_{2}, b_{2}\right)$ such that:

$f\left(a_{1}, b_{1}, 1\right) \neq f\left(a_{2}, b_{2}, 1\right)$,

then there exists an $\alpha \in \mathbb{R}$, such that for all $(a, b, c) \in \mathbb{R} \times \mathbb{R}$ $\{0\} \times \mathbb{R} \backslash\{0\}:$

$f(a, b, c)=\frac{\alpha-a}{b}$

Remark 4.2 In the remainder of the paper $c_{0}$ is fixed and chosen as:

$c_{0}:=[10 \ldots 0]$

The idea on which the proof of Theorem 4.1 is based does unfortunately not apply in the higher order case. However by imposing additional assumptions we obtain the same result. To that end we will explicitly define the function that assigns to every $(A, b, c) \in E$ the characteristic polynomial of the closed-loop system:

Definition 4.3 The function $\chi: E_{r e} \rightarrow \mathbb{R}[X]$ is defined as the function that assigns to every $(A, b) \in E_{r e}$ the characteristic polynomial of $A+b f\left(A, b, c_{0}\right)$.

Saying that $f$ is of the pole-assignment type is equivalent to saying that $\chi$ is constant on $E$, which is exactly what we are going to do after having formulated extra assumptions.

Apart from the ones we already had, we assume additionally: Assumptions:

1. There exists $\left(A_{0}, b_{0}, c_{0}\right) \in E$ such that: $f\left(A_{0}, b_{0}, c_{0}\right)$ and $c_{0}$ are independent vectors.

2. $\forall(A, b, c) \in E: \operatorname{Spec}(A+b f(A, b, c)) \subset \mathbb{R}$.

3. The coefficients of $\chi(A, b, c)$ depend uniformly continuously on $(A, b, c) \in E$

\section{Comment}

1. This assumption is satisfied if $f$ is stabilizing, since not every system can be stabilized by static output feed back. Therefore this assumption is not very restrictive. From now on we will assume that $\left(A_{0}, b_{0}, c_{0}\right)$ has been chosen such that this assumption is satisfied.

2. The assumption that the poles of every closed-loop system are real is a severe restriction. It will appear that this assumption is highly relied upon in the proofs of the forthcoming claims.

3. Uniform continuity of $f$ would be too restrictive since the functions of the pole-assignment type are not absolute continuous. However, uniform continuity of $\chi$ seems reasonable. It enables us to extend $\chi$ on the set of all pairs $(A, b)$. In the sequel we will view $\chi$ as the extended function.

Definition 4.4 For later reference we define:

$G l\left(n, c_{0}\right):=\left\{S \in G l(n) \mid c_{0} S=c_{0}\right\}$

Remark 4.5 In the remainder of this section we choose a fixed triple $\left(A_{0}, b_{0}, c_{0}\right) \in E$ such that the assumptions are satisfied.

Let $\Lambda=\left\{\lambda_{1}, \ldots, \lambda_{m}\right\}$ be the set of eigenvalues of $A_{0}+$ $b_{0} f\left(A_{0}, b_{0}, c_{0}\right)$, not counting their multiplicities.

Let $v$ be an eigenvector of $A_{0}+b_{0} f\left(A_{0}, b_{0}, c_{0}\right), A_{0}+$ $b_{0} f\left(A_{0}, b_{0}, c_{0}\right) v=\lambda v$, say. Since $A_{0}$ and $A_{0}+b_{0} f\left(A_{0}, b_{0}, c_{0}\right)$ have no eigenvalues in common it follows that $f\left(A_{0}, b_{0}, c_{0}\right) v \neq 0$. This implies that we can choose a set of eigenvectors $\left\{v_{1}, \ldots, v_{m}\right\}$ such that $f\left(A_{0}, b_{0}, c_{0}\right) v_{i}=1(i=1, \ldots, m)$.

Define:

$$
\begin{aligned}
\Omega_{i}:=\{ & (A, b) \in E_{r e} \mid \\
& f\left(A, b, c_{0}\right) \text { and } c_{0} \text { dependent vectors, } \\
& \left.f\left(A, b, c_{0}\right) v_{i}=1,\left(A, b, c_{0}\right) \in E\right\}
\end{aligned}
$$

$\Omega:=E \backslash\left(\cup_{i=1}^{m} \Omega_{i}\right) \cap\left\{(A, b) \mid\left(A, b, c_{0}\right) \in E_{r e}\right\}$

We will first analyse $f$ on $\Omega_{i}$.

Lemma 4.6 For all $i: \chi$ is constant on $\Omega_{i}$.

Next we consider $\Omega$.

Lemma 4.7 For every $\left(A_{1}, b_{1}\right)$ in an open and dense subset of $\Omega$.

$\operatorname{Spec}\left(A_{0}+b_{0} f\left(A_{0}, b_{0}, c_{0}\right)\right)=$

$\operatorname{Spec}\left(A_{1}+b_{1} f\left(A_{1}, b_{1}, c_{0}\right)\right)$

Notice that equality in (45) is in the sense of sets. It remains to show that also the multiplicities coincide.

Theorem $4.8 \chi$ is constant on $E$, and hence there exists a monic polynomial $\pi$, of degree $n$ such that for all $(A, b, c) \in E$ :

$f(A, b, c)=-[0 \ldots 01] \cdot\left[b \vdots \ldots \vdots A^{n-1} b\right]^{-1} \pi(A)$

Proof We know already that for $\left(A_{1}, b_{1}, c_{0}\right)$ in a dense subset of $\Omega$, (45) holds. From the uniform continuity of $\chi$ it follows that (45) holds on $\Omega$. As remarked, the equality is only in the sense of sets, the multiplicities could differ from each other. However, for a given set of eigenvalues, there is only a finite number of possibilities for their multiplicities, since the sum of the multiplicities is $n$. As a consequence $\chi$ takes a finite number of values on $\Omega$. Moreover $\chi$ is constant on every $\Omega_{i}$ (Lemma 4.6). Since $\chi$ is uniformly continuous it takes exactly one value and hence is constant on $E_{r e}$.

For arbitrary $c$ a transformation that transforms $c$ into $c_{0}$ can be applied. The transformation property (6) then yields the result.

$\square$

\section{Conclusions}

We have established an answer to the question for which adaptive control problems, zero prediction error implies desired behaviour. We have obtained our results for the general case under the assumption that all the closed-loop poles are real. It is obvious where this assumption is used. Our proofs are based on the intersection of subsets on which the closed-pool poles are constant. If a point of intersection exists, then it follows that the closed-poles of the subsets agree. The central set in this intersection procedure is defined on the basis of a one-dimensional eigenspace of a closed-loop system. For a complex eigenvalue this trick does not work, since the corresponding eigenspace is two-dimensional and as a consequence the dimensions of the spaces of which we want to prove that they intersect, do not sum up to the dimension of the whole space, thus obstructing the genericity of non-empty intersection. This leaves the question whether our results can be extended to the complex case unanswered. Nevertheless we conjecture that this can also be done, and we hope to report on further progress in this matter in the near future. 


\section{References}

1. B.D.O. Anderson And R.M. Johnstone (1985). Global adaptive pole positioning. IEEE Trans. Aut. Contr. AC30, 11-22.

2. K.J. Aström and B. Wittenmark (1973). On self tuning regulators. Automatica 9, 185-199.

3. A. Becker, P.R. Kumar and C.Z. Wei (1985). Adaptive control with the stochastic approximation algorithm: geometry and convergence. IEEE Trans. Aut. Contr. AC$30, \quad 330-338$.

4. H. ElLiot (1982). Direct pole placement with application to nonminimum phase systems. IEEE Trans. Aut. Contr. $A C-27,720,722$.

5. H. Elliott AND W.A. Wolovich (1979). Parameter adaptive identification and control. IEEE Trans. Aut. Control. $A C-24,592-599$.

6. G.C. Goodwin AND K.S. Sin (1981). Adaptive control of nonminimum phase systems. IEEE Trans. Aut. Contr. $A C-26,478-483$.

7. T. Kallath (1980). Linear Systems. Prentice-Hall, Inc., Englewood Cliffs, N.J.

8. G. Kreisselmeier and M.C. Smith (1986). Stable adaptive regulation of arbitrary $n$ th-order plants. IEEE Thans. Aut. Contr. AC-31, 299-305.

9. P. Lancaster (1969). Theory of Matrices. Academic Press, New York.

10. W. Lin, P.R. Kumar and T.I. SEIdMan. (1985) Will the self-tuning approach work for general cost criteria? Systems \& Control Letters 6, 77-86.

11. R. Lozano-Leal and G.C. Goodwin (1985). A globally convergent adaptive pole placement algorithm without a persistency of excitation requirement. IEEE Thans. Aut. Contr. AC-30, 795-798.

12. J.W. Polderman (1987) Adaptive Control and Identification: Conflict or Conflux? Doctoral thesis. Also to appear as a CWI Tract.

13. J.W. Polderman (1988). Adaptive LQ Contral: Conflict of Identification and Control Memorandum No. 718, Department of Applied Mathematics, University of Twente. Also to appear in Journal of Linear Algebra and its Applications (Special Issue on Linear Systems).

14. J.W. Polderman (1989). A State Space Approach to the Problem of Adaptive Pole Assignment. Math. Control Signals Systems 2. 71-94.

15. J.W. Polderman and C. Praagman (1989). The Closed-loop Identification Problem in Indirect Adaptive Control. Memorandom No. ???, Deparment of Applied Mathematics, University of Twente. 